\title{
Superintegrability on the two-dimensional hyperboloid. II
}

\author{
E. G. Kalnins \\ Department of Mathematics and Statistics, University of Waikato, Hamilton, New Zealand
}

W. Miller, Jr.

School of Mathematics, University of Minnesota, Minneapolis, Minnesota 55455

Ye. M. Hakobyan and G. S. Pogosyan

Laboratory of Theoretical Physics, Joint Institute for Nuclear Research,

Dubna, Moscow Region 141980, Russia

(Received 14 December 1998; accepted for publication 7 January 1999)

This work is devoted to the investigation of the quantum mechanical systems on the two-dimensional hyperboloid which admits separation of variables in at least two coordinate systems. Here we consider two potentials introduced in a paper of C. P. Boyer, E. G. Kalnins, and P. Winternitz [J. Math. Phys. 24, 2022 (1983)], which have not yet been studied. We give an example of an interbasis expansion and work out the structure of the quadratic algebra generated by the integrals of motion. (C) 1999 American Institute of Physics. [S0022-2488(99)00505-8]

\section{INTRODUCTION}

Superintegrable systems on the two-dimensional hyperboloid were introduced and developed in Refs. 1-3. In distinction to the cases of two-dimensional Euclidean space and the two-sphere, the classification of superintegrable systems on the hyperboloid is difficult. To date only the four potentials studied in Ref. 3 and two more listed in Ref. 1 are known. In the present paper two potentials are considered, which were constructed in Ref. 1 but have not previously been investigated. These potentials both have only a finite number of bound states. At this point we have treated all the potentials that arise by restriction from Hermitean hyperbolic space. We follow the approach of Ref. 3, which contains an introduction and motivation.

The two-dimensional hyperboloid is characterized via the Cartesian coordinates $\omega_{0}, \omega_{1}, \omega_{2}$ where $\omega_{0}^{2}-\omega_{1}^{2}-\omega_{2}^{2}=1, \omega_{0}>1$. The requirement $\omega_{0}>1$ means that we consider only the upper sheet of the double-sheet hyperboloid. Throughout this paper we will consider the Schrödinger equation on the hyperboloid in the form $(\hbar=m=1)$

$$
H \Psi \equiv\left(-\frac{1}{2} \Delta_{\mathrm{LB}}+V\right) \Psi=E \Psi,
$$

where $V$ is a potential function and the Laplace-Beltrami operator $\Delta_{\mathrm{LB}}$ is written as

$$
\Delta_{\mathrm{LB}}=K_{3}^{2}+K_{2}^{2}-M_{1}^{2} .
$$

Here $K_{3}, K_{2}, M_{1}$ generate the Lie algebra so(2,1) (Refs. 4 and 5):

$$
K_{3}=\omega_{0} \partial_{\omega_{1}}+\omega_{1} \partial_{\omega_{0}}, \quad K_{2}=\omega_{0} \partial_{\omega_{2}}+\omega_{2} \partial_{\omega_{0}}, \quad M_{1}=\omega_{1} \partial_{\omega_{2}}-\omega_{2} \partial_{\omega_{1}}
$$

and

$$
\left[K_{3}, K_{2}\right]=M_{1}, \quad\left[K_{2}, M_{1}\right]=-K_{3}, \quad\left[K_{3}, M_{1}\right]=K_{2} .
$$

The Schrödinger equation (1) for $V=0$ separates in nine coordinate systems. ${ }^{6}$ Introduction of a potential breaks the symmetry and, in general, reduces the number of coordinate systems permitting separability, usually to zero. We consider the following two potentials (see Table I), constructed in Ref. 1, for which (1) is superintegrable. 
TABLE I. Superintegrable potentials.

\begin{tabular}{cl}
\hline \hline Potential $V(\omega)$ & Coordinate system \\
\hline$V_{1}=\frac{\alpha^{2}}{\omega_{2}^{2}}-\frac{\gamma^{2}}{\left(\omega_{0}-\omega_{1}\right)^{2}}+\beta^{2} \frac{\omega_{0}+\omega_{1}}{\left(\omega_{0}-\omega_{1}\right)^{3}}$ & Equidistant \\
& Elliptic-parabolic \\
& Hyperbolic-parabolic \\
& Horicyclic \\
$V_{2}=\frac{\alpha^{2}}{\omega_{2}^{2}}+\gamma^{2} \frac{\omega_{0} \omega_{1}}{\left(\omega_{0}^{2}+\omega_{1}^{2}\right)^{2}}+\left(\alpha^{2}-\beta^{2}\right) \frac{\omega_{0}^{2}-\omega_{1}^{2}}{\left(\omega_{0}^{2}+\omega_{1}^{2}\right)^{2}}$ & \\
\hline \hline
\end{tabular}

Recall that (1) is superintegrable for a given potential $V$ if it is separable simultaneously in at least two coordinate systems.

\section{FIRST POTENTIAL}

The first considered potential is

$$
V_{1}=\frac{\alpha^{2}}{\omega_{2}^{2}}-\frac{\gamma^{2}}{\left(\omega_{0}-\omega_{1}\right)^{2}}+\beta^{2} \frac{\omega_{0}+\omega_{1}}{\left(\omega_{0}-\omega_{1}\right)^{3}},
$$

where $\alpha, \beta, \gamma$ are positive constants. The corresponding Schrödinger equation admits separable solutions in four coordinate systems: equidistant, elliptic-parabolic, hyperbolic-parabolic, and horicyclic.

\section{A. Solutions of the Schrödinger equation}

\section{Equidistant coordinates}

In this coordinate system

$$
\omega_{0}=\cosh \tau_{1} \cosh \tau_{2}, \quad \omega_{1}=\cosh \tau_{1} \sinh \tau_{2}, \quad \omega_{2}=\sinh \tau_{1}
$$

$\left[\tau_{1}, \tau_{2} \in(-\infty, \infty)\right]$, the potential $V_{1}$ has the form

$$
V_{1}\left(\tau_{1}, \tau_{2}\right)=\frac{\alpha^{2}}{\sinh ^{2} \tau_{1}}+\frac{1}{\cosh ^{2} \tau_{1}} \frac{\beta^{2}-\gamma^{2}\left(\cosh \tau_{2}-\sinh \tau_{2}\right)^{2}}{\left(\cosh \tau_{2}-\sinh \tau_{2}\right)^{4}} .
$$

After putting

$$
\Psi\left(\tau_{1}, \tau_{2}\right)=\left(\cosh \tau_{1}\right)^{-1 / 2} S_{1}\left(\tau_{1}\right) S_{1}\left(\tau_{2}\right),
$$

we come to the system of equations:

$$
\begin{gathered}
\frac{d^{2} S_{2}}{d \tau_{2}^{2}}+\left[-\mu^{2}-2 \beta^{2} e^{4 \tau_{2}}+2 \gamma^{2} e^{2 \tau_{2}}\right] S_{2}=0, \\
\frac{d^{2} S_{1}}{d \tau_{1}^{2}}+\left[\left(2 E-\frac{1}{4}\right)+\frac{\mu^{2}-\frac{1}{4}}{\cosh ^{2} \tau_{1}}-\frac{2 \alpha^{2}}{\sinh ^{2} \tau_{1}}\right] S_{1}=0,
\end{gathered}
$$


where $\mu$ is the equidistant separation constant. The first equation (8) could be considered as a one-dimensional Schrödinger equation for the Morse potential ${ }^{7}$ and the orthonormalized solution is given by the expression

$$
\begin{aligned}
S_{2}\left(\tau_{2}\right) \equiv S_{m}^{(\beta, \mu)}(z) & =\sqrt{\frac{2 \mu \Gamma(m+\mu+1)}{m ! \Gamma^{2}(\mu+1)}} e^{-z / 2} z^{\mu / 2}{ }_{2} F_{1}(-m, \mu+1 ; z) \\
& =\sqrt{\frac{2 \mu m !}{\Gamma(m+\mu+1)}} e^{-z / 2} z^{\mu / 2} L_{m}^{\mu}(z), \quad z=\sqrt{2} \beta e^{2 \tau_{2}}
\end{aligned}
$$

where $L_{m}^{\mu}(z)$ are the Laguerre polynomials. ${ }^{8}$ The separation constant is quantized as

$$
\mu=-2 m-1+\frac{\gamma^{2}}{\sqrt{2} \beta}, \quad 0 \leqslant m \leqslant\left[\frac{1}{2}\left(\frac{\gamma^{2}}{\sqrt{2} \beta}-1\right)\right] .
$$

The second equation (9) represents the modified Pöschl-Teller equation. ${ }^{3,9}$ The orthonormalized wave function is given by

$$
\begin{aligned}
S_{1}\left(\tau_{1}\right) \equiv S_{n}^{(\alpha, \mu)}\left(\tau_{1}\right)= & \sqrt{\frac{2\left(\mu-\sqrt{2 \alpha^{2}+1 / 4}-2 n-1\right) \Gamma(\mu-n) n !}{\Gamma\left(\mu-\sqrt{2 \alpha^{2}+1 / 4}-n\right) \Gamma\left(1+n+\sqrt{2 \alpha^{2}+1 / 4}\right)}} \\
& \times\left(\sinh \tau_{1}\right)^{1 / 2+\sqrt{2 \alpha^{2}+1 / 4}}\left(\cosh \tau_{1}\right)^{1 / 2-\mu} P_{n}^{\left(\sqrt{2 \alpha^{2}+1 / 4},-\mu\right)}\left(\cosh 2 \tau_{1}\right),
\end{aligned}
$$

with $n=0,1, \ldots,\left[\frac{1}{2}\left(\mu-1-\sqrt{2 \alpha^{2}+\frac{1}{4}}\right)\right]$, where $P_{n}^{(\alpha, \beta)}(x)$ is the Jacobi polynomial. ${ }^{8}$ The quantized energy is

$$
E_{N}=-\frac{1}{2}\left(\mu-\sqrt{2 \alpha^{2}+\frac{1}{4}}-2 n-1\right)^{2}+\frac{1}{8}=-\frac{1}{2}\left(2 N+2+\sqrt{2 \alpha^{2}+\frac{1}{4}}-\frac{\gamma^{2}}{\sqrt{2} \beta}\right)^{2}+\frac{1}{8},
$$

where $N=m+n$ is the principal quantum number and the bound states occur for

$$
0 \leqslant N \leqslant\left[\frac{1}{2}\left(\frac{\gamma^{2}}{\sqrt{2} \beta}-\sqrt{2 \alpha^{2}+1 / 4}-2\right)\right] .
$$

The orthonormalized total wave function $\Psi_{n m}\left(\tau_{1}, \tau_{2}\right)$ is given by (7), (10), and (12).

The symmetry operator describing this coordinate system is

$$
\begin{aligned}
L_{1} \Psi_{n m}\left(\tau_{1}, \tau_{2}\right) & \equiv\left[K_{3}^{2}-2 \beta^{2}\left(\frac{\omega_{0}+\omega_{1}}{\omega_{0}-\omega_{1}}\right)^{2}+2 \gamma^{2} \frac{\omega_{0}+\omega_{1}}{\omega_{0}-\omega_{1}}\right] \Psi_{n m}\left(\tau_{1}, \tau_{2}\right) \\
& =\left(-2 m-1+\frac{\gamma^{2}}{\sqrt{2} \beta}\right)^{2} \Psi_{n m}\left(\tau_{1}, \tau_{2}\right) .
\end{aligned}
$$

\section{Horicyclic coordinates}

In the horicyclic coordinates,

$$
\omega_{0}=\frac{x^{2}+y^{2}+1}{2 y}, \quad \omega_{1}=\frac{x^{2}+y^{2}-1}{2 y}, \quad \omega_{2}=\frac{x}{y}
$$

$[y>0, x \in(-\infty, \infty)]$, the potential $V_{1}$ is 


$$
V_{1}(x, y)=y^{2}\left[\frac{\alpha^{2}}{x^{2}}+\beta^{2}\left(x^{2}+y^{2}\right)-\gamma^{2}\right]
$$

and the Schrödinger equation has the following form:

$$
-\frac{1}{2} y^{2}\left[\frac{\partial^{2}}{\partial x^{2}}-\frac{2 \alpha^{2}}{x^{2}}-2 \beta^{2} x^{2}+\frac{\partial^{2}}{\partial y^{2}}-2 \beta^{2} y^{2}+2 \gamma^{2}\right] \Psi(x, y)=E \Psi(x, y) .
$$

Via putting

$$
\Psi(x, y)=\psi_{1}(x) \psi_{2}(y),
$$

it admits a separation

$$
\begin{aligned}
& \frac{d^{2} \psi_{1}}{d x^{2}}+2\left[\gamma^{2}\left(\lambda_{1}+1\right)-\beta^{2} x^{2}-\frac{\alpha^{2}}{x^{2}}\right] \psi_{1}=0, \\
& \frac{d^{2} \psi_{2}}{d y^{2}}+2\left[\gamma^{2}\left(\lambda_{2}-1\right)-\beta^{2} y^{2}+\frac{E}{y^{2}}\right] \psi_{2}=0,
\end{aligned}
$$

where $\lambda_{1}$ and $\lambda_{2}$ are the horicyclic separation constants with the relation $\lambda_{1}+\lambda_{2}=1$.

The orthonormalized solutions of the equations (20) and (21) for $\left(-2 E+\frac{1}{4}\right)>0$ are

$$
\begin{gathered}
\psi_{1}(x) \equiv \psi_{n_{1}}^{(\alpha, \beta)}(x)=\sqrt{\frac{n_{1} !(\sqrt{2} \beta)^{1 / 2}}{\Gamma\left(n_{1}+\sqrt{2 \alpha^{2}+\frac{1}{4}}+1\right)}} e^{-\beta x^{2} / \sqrt{2}}\left(\sqrt{\sqrt{2} \beta x^{2}}\right)^{1 / 2}+\sqrt{2 \alpha^{2}+1 / 4} L_{n_{1}}^{\sqrt{2 \alpha^{2}+1 / 4}}\left(\sqrt{2} \beta x^{2}\right), \\
\psi_{2}(y) \equiv \psi_{n_{2}}^{(\gamma, \beta)}(y) \\
=\sqrt{\frac{n_{2} !(\sqrt{2} \beta)^{1 / 2}}{\Gamma\left(n_{2}+\sqrt{-2 E+\frac{1}{4}}+1\right)}} e^{-\beta y^{2} / \sqrt{2}}\left(\sqrt{\sqrt{2} \beta y^{2}}\right)^{1 / 2+\sqrt{-2 E+1 / 4}} L_{n_{2}}^{\sqrt{-2 E+1 / 4}}\left(\sqrt{2} \beta y^{2}\right) .
\end{gathered}
$$

The separation constants $\lambda_{1}, \lambda_{2}$ are quantized as

$$
\lambda_{1}=\frac{\sqrt{2} \beta}{\gamma^{2}}\left(2 n_{1}+\sqrt{2 \alpha^{2}+\frac{1}{4}}+1\right)-1 ; \quad \lambda_{2}=\frac{\sqrt{2} \beta}{\gamma^{2}}\left(2 n_{2}+\sqrt{-2 E+\frac{1}{4}}+1\right)+1,
$$

and according to the relation $\lambda_{1}+\lambda_{2}=1$, we come to the energy spectrum as in (13). The operator characterizing the separation in horicyclic coordinates is

$$
\begin{aligned}
L_{2} \Psi_{n_{1} n_{2}}(x, y) & \equiv\left[\left(K_{2}-M_{1}\right)^{2}-\frac{2 \beta^{2} \omega_{2}^{2}}{\left(\omega_{0}-\omega_{1}\right)^{2}}-\frac{2 \alpha^{2}\left(\omega_{0}-\omega_{1}\right)^{2}}{\omega_{2}^{2}}+2 \gamma^{2}\right] \Psi_{n_{1} n_{2}}(x, y) \\
& =-\left[2 \sqrt{2} \beta\left(2 n_{1}+\sqrt{2 \alpha^{2}+\frac{1}{4}}+1\right)+2 \gamma^{2}\right] \Psi_{n_{1} n_{2}}(x, y) .
\end{aligned}
$$

\section{Elliptic-parabolic coordinates}

In this coordinate system,

$$
\omega_{0}=\frac{\cosh ^{2} a+\cos ^{2} \theta}{2 \cosh a \cos \theta}, \quad \omega_{1}=\frac{\sinh ^{2} a-\sin ^{2} \theta}{2 \cosh a \cos \theta}, \quad \omega_{2}=\tanh a \tan \theta
$$


$[a>0, \theta \in(-\pi / 2, \pi / 2)]$, the potential $V_{1}$ has the form

$$
\begin{aligned}
V_{1}(a, \theta)= & \frac{\cosh ^{2} a \cos ^{2} \theta}{\cosh ^{2} a-\cos ^{2} \theta}\left[\beta^{2}\left(\cosh ^{2} a \sinh ^{2} a+\cos ^{2} \theta \sin ^{2} \theta\right)\right. \\
& \left.-\gamma^{2}\left(\cosh ^{2} a-\cos ^{2} \theta\right)+\alpha^{2}\left(\frac{1}{\sinh ^{2} a}+\frac{1}{\sin ^{2} \theta}\right)\right] .
\end{aligned}
$$

The Schrödinger equation is

$$
\begin{aligned}
-\frac{1}{2} & \frac{\cosh ^{2} a \cos ^{2} \theta}{\cosh ^{2} a-\cos ^{2} \theta}\left[\frac{\partial^{2}}{\partial a^{2}}-2 \beta^{2} \cosh ^{2} a \sinh ^{2} a+2 \gamma^{2} \cosh ^{2} a-\frac{2 \alpha^{2}}{\sinh ^{2} a}\right. \\
+ & \left.\frac{\partial^{2}}{\partial \theta^{2}}-2 \beta^{2} \cos ^{2} \theta \sin ^{2} \theta-2 \gamma^{2} \cos ^{2} \theta-\frac{2 \alpha^{2}}{\sin ^{2} \theta}\right] \Psi(a, \theta)=E \Psi(a, \theta) .
\end{aligned}
$$

Putting for the wave function $\Psi(a, \theta)=S(a) S(\theta)$, after separation of variables we get two identical equations:

$$
\frac{d^{2} S(\rho)}{d \rho^{2}}+\left[\lambda-2 \beta^{2} \cosh ^{2} \rho \sinh ^{2} \rho+2 \gamma^{2} \cosh ^{2} \rho-\frac{2 \alpha^{2}}{\sinh ^{2} \rho}-\frac{2 E}{\cosh ^{2} \rho}\right] S(\rho)=0,
$$

where $\lambda$ is the elliptic-parabolic separation constant and $\rho \equiv a, i \theta$. After changing the variables $x=\cosh ^{2} \rho$ in Eq. (29), we obtain

$$
4 x(x-1) \frac{d^{2} S}{d x^{2}}+2(2 x-1) \frac{d S}{d x}+\left[\lambda-2 \beta^{2} x(x-1)+2 \gamma^{2} x-\frac{2 \alpha^{2}}{x-1}-\frac{2 E}{x}\right] S=0 .
$$

Thus the region $x \in[1, \infty]$ in Eq. (30) belongs to the wave function $S(a)$ and $x \in[0,1]$ to the wave function $S(\theta)$. Putting

$$
S(x)=(x-1)^{s} x^{t} e^{-\beta x / \sqrt{2}} G(x),
$$

where

$$
s=\frac{1}{4}+\frac{1}{\sqrt{2}} \sqrt{\alpha^{2}+\frac{1}{8}}, \quad t=\frac{1}{4}+\frac{1}{\sqrt{2}} \sqrt{-E+\frac{1}{8}},
$$

we get

$$
\begin{aligned}
\frac{d^{2} G}{d x^{2}}+ & \frac{1}{2}\left[\frac{1+4 t}{x}+\frac{1+4 s}{x-1}-\frac{4 \beta}{\sqrt{2}}\right] \frac{d G}{d x} \\
& +\frac{1}{4}\left\{\frac{\left[2 \gamma^{2}-4 \beta(1+2(t+s)) / \sqrt{2}\right] x+\nu+\sqrt{2} \beta(1+4 t)+4(t+s)^{2}}{x(x-1)}\right\} G=0 .
\end{aligned}
$$

If we now substitute

$$
G(x)=\prod_{i=1}^{N}\left(x-\theta_{i}\right)
$$

and take into account (32), we find that $\theta_{i}$ satisfies the equation 


$$
2 \theta_{i}\left(1-\theta_{i}\right)\left(\sum_{\substack{k=1 \\ k \neq i}}^{N} \frac{1}{\theta_{k}-\theta_{i}}+\frac{\beta}{\sqrt{2}}\right)+2\left(1-\theta_{i}\right) N+\frac{\sqrt{2} \gamma^{2}}{4 \beta} \theta_{i}+\frac{\gamma^{2}}{\sqrt{2} \beta}+\sqrt{2 \alpha^{2}+\frac{1}{4}}+1=0 .
$$

The quantization for the energy is given via

$$
\sqrt{-2 E+\frac{1}{4}}+\sqrt{2 \alpha^{2}+\frac{1}{4}}+2 N+2-\frac{\gamma^{2}}{\sqrt{2} \beta}=0,
$$

and we obtain the expression (13). The separation constant $\lambda$ is

$$
\lambda=\frac{8 \beta}{\sqrt{2}} \sum_{i=1}^{N} \theta_{i}-\left(\frac{\gamma^{2}}{\sqrt{2} \beta}-1\right)^{2}+\frac{4 \beta}{\sqrt{2}}\left(1+\sqrt{2 \alpha^{2}+\frac{1}{4}}\right)-2 \gamma^{2} .
$$

Thus the total solution $\Psi(a, \theta)$ is represented as

$$
\begin{aligned}
\Psi_{N p q}(a, \theta)= & S_{N p}(a) S_{N q}(\theta) \\
= & (\sinh a \sin \theta)^{1 / 2+\sqrt{2 \alpha^{2}+1 / 4}}(\cosh a \cos \theta)^{\gamma^{2} / \sqrt{2} \beta-\sqrt{2 \alpha^{2}+1 / 4}-2 N-3 / 2} \\
& \times \exp \left\{-\frac{\beta}{\sqrt{2}}\left(\cosh ^{2} a+\cos ^{2} \theta\right)\right\} \prod_{i=1}^{N}\left(\cosh ^{2} a-\theta_{i}\right)\left(\cos ^{2} \theta-\theta_{i}\right),
\end{aligned}
$$

where $p$ and $q$ are the number of zeros for the wave functions $S(a)$ and $S(\theta)$ in the regions $[0,1]$, $[1, \infty]$ correspondingly, and the total number of zeros is $N=p+q$.

Eliminating the energy $E$ from Eq. (30), we see that the additional integral of motion here is

$$
\begin{aligned}
L_{3} \Psi_{N p q}(a, \theta)= & \frac{1}{\cos ^{2} \theta-\cosh ^{2} a}\left\{\cosh ^{2} a \frac{\partial^{2}}{\partial a^{2}}+\cos ^{2} \theta \frac{\partial^{2}}{\partial \theta^{2}}-2 \beta^{2}\left(\cosh ^{4} a \sinh ^{2} a+\cos ^{4} \theta \sin ^{2} \theta\right)\right. \\
& \left.+2 \gamma^{2}\left(\cosh ^{4} a-\cos ^{4} \theta\right)-2 \alpha^{2}\left(\operatorname{coth}^{2} a-\cot ^{2} \theta\right)\right\} \Psi_{N p q}(a, \theta) \\
= & \left\{-\left(K_{2}-M_{1}\right)^{2}-K_{3}^{2}+2 \beta^{2} \frac{\left(w_{0}+w_{1}\right)^{2}+w_{2}^{2}}{\left(w_{0}-w_{1}\right)^{2}}+2 \alpha^{2}\left(\frac{w_{0}-w_{1}}{w_{2}}\right)^{2}\right. \\
& \left.-4 \gamma^{2} \frac{w_{0}}{w_{0}-w_{1}}\right\} \Psi_{N p q}(a, \theta) \\
= & \lambda \Psi_{N p q}(a, \theta) .
\end{aligned}
$$

\section{Hyperbolic-parabolic coordinates}

In this coordinate system,

$$
\omega_{0}=\frac{\cosh ^{2} b+\cos ^{2} \theta}{2 \sinh b \sin \theta}, \quad \omega_{1}=\frac{\sinh ^{2} b-\sin ^{2} \theta}{2 \sinh b \sin \theta}, \quad \omega_{2}=\operatorname{coth} b \cot \theta
$$

$[b>0, \theta \in(-\pi / 2, \pi / 2)]$, the potential $V_{1}$ has the form

$$
\begin{aligned}
V_{1}(b, \theta)= & \frac{\sinh ^{2} b \sin ^{2} \theta}{\sinh ^{2} b+\sin ^{2} \theta}\left[\beta^{2}\left(\sinh ^{2} b \cosh ^{2} b+\sin ^{2} \theta \cos ^{2} \theta\right)\right. \\
& \left.-\gamma^{2}\left(\sinh ^{2} b+\sin ^{2} \theta\right)+\alpha^{2}\left(\frac{1}{\cos ^{2} \theta}-\frac{1}{\cosh ^{2} b}\right)\right]
\end{aligned}
$$


The Schrödinger equation is

$$
\begin{aligned}
-\frac{1}{2} & \frac{\sinh ^{2} b \sin ^{2} \theta}{\sinh ^{2} b+\sin ^{2} \theta}\left[\frac{\partial^{2}}{\partial b^{2}}-2 \beta^{2} \sinh ^{2} b \cosh ^{2} b+2 \gamma^{2} \sinh ^{2} b+\frac{2 \alpha^{2}}{\cosh ^{2} b}\right. \\
+ & \left.\frac{\partial^{2}}{\partial \theta^{2}}-2 \beta^{2} \sin ^{2} \theta \cos ^{2} \theta+2 \gamma^{2} \sin ^{2} \theta-\frac{2 \alpha^{2}}{\cos ^{2} \theta}\right] \Psi(b, \theta)=E \Psi(b, \theta) .
\end{aligned}
$$

Putting for the wave function $\Psi(b, \theta)=S(b) S(\theta)$, after separation of variables we get two identical equations:

$$
\frac{d^{2} S(\rho)}{d \rho^{2}}+2\left[\frac{\tau}{2}-\beta^{2} \sinh ^{2} \rho \cosh ^{2} \rho+\gamma^{2} \sinh ^{2} \rho+\frac{\alpha^{2}}{\cosh ^{2} \rho}+\frac{E}{\sinh ^{2} \rho}\right] S(\rho)=0,
$$

where $\tau$ is the hyperbolic-parabolic separation constant and $\rho \equiv b, i \theta$. After changing the variables $x=\sinh ^{2} \rho$ in Eq. (43), we come to the equation

$$
4 x(x+1) \frac{d^{2} S}{d x^{2}}+2(2 x+1) \frac{d S}{d x}+\left[\tau-2 \beta^{2} x(x+1)+2 \gamma^{2} x+\frac{2 \alpha^{2}}{x+1}+\frac{2 E}{x}\right] S=0 .
$$

Choosing

$$
P(x)=(1+x)^{s} x^{t} e^{-\beta x / \sqrt{2}} \prod_{i=1}^{N}\left(x-\theta_{i}\right)
$$

where $t$ and $s$ are given by the formulas (32), we obtain the energy spectrum (36). Here $\theta_{i}$ satisfies the equations

$$
2 \theta_{i}\left(1+\theta_{i}\right)\left(\sum_{\substack{k=1 \\ k \neq i}}^{N} \frac{1}{\theta_{i}-\theta_{k}}-\frac{\beta}{\sqrt{2}}\right)-2\left(1+\theta_{i}\right) N+\frac{\sqrt{2} \gamma^{2}}{4 \beta} \theta_{i}+\frac{\gamma^{2}}{\sqrt{2} \beta}-\sqrt{2 \alpha^{2}+\frac{1}{4}}-1=0
$$

The separation constant $\tau$ is

$$
\tau=\frac{8 \beta}{\sqrt{2}} \sum_{i=1}^{N} \theta_{i}-\left(\frac{\gamma^{2}}{\sqrt{2} \beta}-1\right)^{2}-\frac{4 \beta}{\sqrt{2}}\left(1+\sqrt{2 \alpha^{2}+\frac{1}{4}}\right)+2 \gamma^{2}
$$

so the total solution $\Psi(b, \theta)$ is represented as

$$
\begin{aligned}
\Psi_{N l k}(b, \theta)= & S_{N l}(b) S_{N k}(\theta) \\
= & (\cosh b \cos \theta)^{1 / 2+\sqrt{2 \alpha^{2}+1 / 4}}(\sinh b \sin \theta)^{\gamma^{2} / \sqrt{2} \beta-\sqrt{2 \alpha^{2}+1 / 4}-2 N-3 / 2} \\
& \cdot \exp \left\{-\frac{\beta}{\sqrt{2}}\left(\sinh ^{2} b-\sin ^{2} \theta\right)\right\} \prod_{i=1}^{N}\left(\sinh ^{2} b-\theta_{i}\right)\left(\sin ^{2} \theta+\theta_{i}\right) .
\end{aligned}
$$

The total number of zeros is $N$, and $k$ of them are located in the interval $[-1,0]$ and $l$ are in $[0, \infty]$.

Each solution $\Psi_{N l k}(b, \theta)$ satisfies the eigenvalue equation 


$$
\begin{aligned}
L_{4} \Psi_{N l k}(b, \theta)= & -\frac{1}{\sin ^{2} b+\sin ^{2} \theta}\left\{\sinh ^{2} b \frac{\partial^{2}}{\partial b^{2}}-\sin ^{2} \theta \frac{\partial^{2}}{\partial \theta^{2}}-2 \beta^{2}\left(\cosh ^{2} b \sinh ^{4} b-\cos ^{2} \theta \sin ^{4} \theta\right)\right. \\
& \left.+2 \gamma^{2}\left(\sinh ^{4} b-\sin ^{4} \theta\right)+2 \alpha^{2}\left(\tanh ^{2} b+\tan ^{2} \theta\right)\right\} \Psi_{N l k}(b, \theta) \\
= & \left\{\left(K_{2}-M_{1}\right)^{2}-K_{3}^{2}+2 \beta^{2} \frac{\left(w_{0}+w_{1}\right)^{2}-w_{2}^{2}}{\left(w_{0}-w_{1}\right)^{2}}-2 \alpha^{2}\left(\frac{w_{0}-w_{1}}{w_{2}}\right)^{2}\right. \\
& \left.-4 \gamma^{2} \frac{w_{1}}{w_{0}-w_{1}}\right\} \Psi_{N l k}(b, \theta)=\tau \Psi_{N l k}(b, \theta) .
\end{aligned}
$$

\section{B. Algebra}

Among the operators $\left\{L_{1}, L_{2}, L_{3}, L_{4}\right\}$, corresponding to the four separable coordinate systems, only two are independent, as

$$
L_{3}=-L_{2}-L_{1}, \quad L_{4}=L_{2}-L_{1} .
$$

Consider the operators $N_{1}, N_{2}$, and $R$ where

$$
\begin{aligned}
& N_{1}=\widetilde{L}_{2}=L_{1}, \quad N_{2}=\widetilde{L}_{1}=L_{2}-2 \gamma^{2}, \\
& R \equiv\left[N_{1}, N_{2}\right]= 2\left\{K_{3},\left\{K_{2}, M_{1}\right\}\right\}-2\left\{K_{3}, K_{2}^{2}\right\}-2\left\{K_{3}, M_{1}^{2}\right\} \\
&+8\left[\alpha^{2}\left(\frac{\omega_{0}-\omega_{1}}{\omega_{2}}\right)^{2}+\beta^{2}\left(\frac{\omega_{2}}{\omega_{0}-\omega_{1}}\right)^{2}\right] K_{3}+\frac{16 \beta^{2} \omega_{2}}{\left(\omega_{0}-\omega_{1}\right)^{2}}\left(\omega_{0} K_{2}-\omega_{1} M_{1}\right) \\
&+\frac{8 \gamma^{2} \omega_{2}}{\omega_{0}-\omega_{1}}\left(M_{1}-K_{2}\right)-4\left[\gamma^{2}+2 \alpha^{2}\left(\frac{\omega_{0}-\omega_{1}}{\omega_{2}}\right)^{2}-2 \beta^{2} \frac{1+2 \omega_{2}^{2}}{\left(\omega_{0}-\omega_{1}\right)^{2}}\right] .
\end{aligned}
$$

We have

$$
\begin{gathered}
{\left[R, N_{2}\right]=-8 N_{2}^{2}-64 \beta^{2} H-16 \gamma^{2} N_{2}-32 \beta^{2} N_{1}+16 \beta^{2}\left(4 \alpha^{2}-1\right),} \\
{\left[R, N_{1}\right]=4\left\{N_{1}, N_{2}\right\}+32 \gamma^{2} H-16 N_{2}+16 \gamma^{2} N_{1}+16 \gamma^{2}(2 \alpha-1),} \\
R^{2}=\frac{8}{3}\left\{N_{2}, N_{2}, L_{1}\right\}-\frac{176}{3} N_{2}^{2}+32 \beta^{2} N_{1}^{2}+128 \beta^{2} H^{2}+64 \gamma^{2} H N_{2}+128 \beta^{2} H N_{1} \\
+16 \gamma^{2}\left\{N_{1}, N_{2}\right\}+\left(\frac{128}{3}+256 \alpha^{2} \beta^{2}\right) H+\left(64 \alpha^{2} \gamma^{2}-\frac{352}{3} \gamma^{2}\right) N_{2}+\left(\frac{352}{3}-128 \alpha^{2} \beta^{2}\right) N_{1} \\
+\left(128 \alpha^{4} \beta^{2}+128 \gamma^{4} \alpha^{2}-\frac{128}{3} \alpha^{2} \beta^{2}-\frac{64}{3} \beta^{2}-48 \gamma^{2}\right),
\end{gathered}
$$

where $\{A, B\}=A B+B A$ and

$$
\{A, B, C\}=A B C+A C B+B C A+B A C+C A B+C B A .
$$

The integrals of motion $N_{1}, N_{2}$, and $H$ generate a quadratic algebra.

\section{Interbasis expansion}

For a fixed value of energy, we can write the equidistant wave function (7) in terms of the horicyclic ones (19) as

$$
\Psi_{n_{1} n_{2}}(x, y)=\sum_{m=0}^{n_{1}+n_{2}} W_{n_{1} n_{2}}^{n m}(\alpha, \beta, \gamma) \Psi_{n m}(a, b),
$$


where $n_{1}+n_{2}=n+m$. The connection between the equidistant $(a, b)$ and horicyclic $(x, y)$ coordinates is

$$
x=e^{b} \tanh a, \quad y=e^{b} \frac{1}{\cosh a} .
$$

Going over to the horicyclic coordinates on the left side of expansion (54), then considering the limit $b \rightarrow \infty$ and using the asymptotic formula for Laguerre polynomials ${ }^{8}$

$$
\lim _{x \rightarrow \infty} L_{n}^{\alpha}(x) \rightarrow(-1)^{n} \frac{x^{n}}{n !},
$$

we see that dependence on $b$ cancels on both sides of (54). Now, using the orthogonality condition for the angular wave functions (12), we find the following expression for the interbasis coefficients $W_{n_{1} n_{2}}^{n m}$ :

$$
W_{n_{1} n_{2}}^{n m}=(-1)^{n} \sqrt{\frac{m ! n ! \sqrt{2} \beta(\mu-d-2 n-1) \Gamma(\mu+m+1) \Gamma(\mu-n)}{n_{1} ! n_{2} ! \mu \Gamma\left(n_{1}+d+1\right) \Gamma\left(n_{2}+d+1\right) \Gamma(n+d+1) \Gamma(\mu-d-n)}} B_{n_{1} n_{2}}^{n m},
$$

where

$$
B_{n_{1} n_{2}}^{n m}=\int_{-\infty}^{+\infty}(\sinh a)^{1+2 d+2 n_{1}}(\cosh a)^{1-2 \mu-2 m} P_{n}^{(d,-\mu)}(\cosh 2 a) d a
$$

and $d=\sqrt{2 \alpha^{2}+\frac{1}{4}}$. The integral $B_{n_{1}, n_{2}}^{n m}$ can be evaluated by expressing the Jacobi polynomial through the hypergeometric function ${ }_{2} F_{1}:{ }^{8}$

$$
P_{n}^{(\alpha, \beta)}(x)=(-1)^{n} \frac{\Gamma(n+\beta+1)}{\Gamma(\beta+1) n !}{ }_{2} F_{1}\left(\begin{array}{c|c}
-n, n+\alpha+\beta+1 & 1+x \\
\beta+1
\end{array}\right) .
$$

Representing the function ${ }_{2} F_{1}$ as a series we come to a sum of integrals, each of which can be calculated by using the formula ${ }^{8}$

$$
\int_{0}^{+\infty}(\sinh \tau)^{\alpha}(\cosh \tau)^{-\beta} d \tau=\frac{1}{2} B\left(\frac{1+\alpha}{2}, \frac{\beta-\alpha}{2}\right) \quad[\operatorname{Re} \alpha>-1, \operatorname{Re}(\alpha-\beta)<0]
$$

We thus obtain

$$
\begin{aligned}
W_{n_{1} n_{2}}^{n m}= & \frac{(-1)^{n}}{2} \sqrt{\frac{m ! \sqrt{2} \beta(\mu-d-2 n-1)(\mu+m) \Gamma\left(n_{1}+d+1\right)}{n ! n_{1} ! n_{2} ! \mu \Gamma\left(n_{2}+d+1\right) \Gamma(n+d+1) \Gamma(\mu-n-d)}} \\
& \times \frac{\Gamma(\mu) \Gamma\left(\mu+m-d-n_{1}-1\right)}{\sqrt{\Gamma(\mu-n) \Gamma(\mu+m)}}{ }_{3} F_{2}\left(\begin{array}{c}
-n, n+d-\mu+1,1-\mu-m \\
1-\mu, 2+n_{1}+d-\mu-m
\end{array} \mid 1\right) .
\end{aligned}
$$

Alternatively, by using the formula ${ }^{10}$ for the Hahn polynomials $h_{n}^{(\alpha, \beta)}(x, N)$,

$$
h_{n}^{(\alpha, \beta)}(x, N)=\frac{(-1)^{n} \Gamma(N) \Gamma(\beta+n+1)}{n ! \Gamma(N-n) \Gamma(\beta+1)}{ }_{3} F_{2}\left(\begin{array}{l}
-n ; \alpha+\beta+n+1 ;-x \\
\beta+1 ; 1-N
\end{array} \mid 1\right),
$$

we obtain the following expression for the expansion coefficients: 


$$
\begin{aligned}
W_{n_{1} n_{2}}^{n m}= & \frac{(-1)^{n}}{2} \sqrt{\frac{m ! n ! \sqrt{2} \beta(\mu-d-2 n-1)(\mu+m)}{n_{1} ! n_{2} ! \mu \Gamma(n+d+1) \Gamma(\mu-n-d)}} \cdot \sqrt{\frac{\Gamma\left(n_{1}+d+1\right) \Gamma(\mu-n)}{\Gamma\left(n_{2}+d+1\right) \Gamma(\mu+m)}} \\
& \times \Gamma\left(\mu+m-d-n_{1}-n-1\right) \cdot h_{n}^{(d,-\mu)}\left(\mu+m+1, \mu+m-d-n_{1}-1\right),
\end{aligned}
$$

in terms of Hahn polynomials.

\section{SECOND POTENTIAL}

The second considered potential is

$$
V_{2}=\frac{\alpha^{2}}{\omega_{2}^{2}}+\gamma^{2} \frac{\omega_{0} \omega_{1}}{\left(\omega_{0}^{2}+\omega_{1}^{2}\right)^{2}}+\left(\alpha^{2}-\beta^{2}\right) \frac{\omega_{0}^{2}-\omega_{1}^{2}}{\left(\omega_{0}^{2}+\omega_{1}^{2}\right)^{2}},
$$

where $\alpha, \beta$, and $\gamma$ are positive constants. The corresponding Schrödinger equation admits separable solutions in two coordinate systems: equidistant and semi-hyperbolic.

\section{A. Solutions of the Schrödinger equation}

\section{Equidistant coordinates}

In this coordinate system,

$$
\omega_{0}=\cosh \tau_{1} \cosh \tau_{2}, \quad \omega_{1}=\cosh \tau_{1} \sinh \tau_{2}, \quad \omega_{2}=\sinh \tau_{1}
$$

$\left[\tau_{1}, \tau_{2} \in(-\infty, \infty)\right]$, the potential $V_{2}$ has the form

$$
V_{2}\left(\tau_{1}, \tau_{2}\right)=-\frac{\alpha^{2}}{\sinh ^{2} \tau_{1}}+\frac{1}{\cosh ^{2} \tau_{1}} \frac{\alpha^{2}-\beta^{2}+\gamma^{2} \cosh \tau_{2} \sinh \tau_{2}}{\left(\cosh ^{2} \tau_{2}+\sinh ^{2} \tau_{2}\right)^{2}} .
$$

After putting

$$
\Psi\left(\tau_{1}, \tau_{2}\right)=\left(\cosh \tau_{1}\right)^{-1 / 2} Z\left(\tau_{1}\right) S\left(\tau_{2}\right)
$$

we arrive at two equations:

$$
\begin{gathered}
\frac{d^{2} S}{d \tau_{2}^{2}}+\left[-\mu^{2}-\frac{2\left(\alpha^{2}-\beta^{2}\right)+\gamma^{2} \sinh \left(2 \tau_{2}\right)}{\cosh ^{2}\left(2 \tau_{2}\right)}\right] S=0, \\
\frac{d^{2} Z}{d \tau_{1}^{2}}+\left[2 E-\frac{1}{4}+\frac{\mu^{2}-\frac{1}{4}}{\cosh ^{2} \tau_{1}}-\frac{2 \alpha^{2}}{\sinh ^{2} \tau_{1}}\right] Z=0,
\end{gathered}
$$

where $\mu$ is the equidistant separation constant.

Let us consider the first equation (68). The substitution $x=\sinh 2 \tau_{2}$ transforms this equation to

$$
4\left(1+x^{2}\right) \frac{d^{2} S}{d x^{2}}+4 x \frac{d S}{d x}+\left[-\mu^{2}+\frac{2\left(\beta^{2}-\alpha^{2}\right)-\gamma^{2} x}{\left(1+x^{2}\right)}\right] S=0,
$$

where the physical region is $x \in(-\infty, \infty)$. The equation (68) has three regular singularities in the points $x=-i, i, \infty$ and may be solved in term of hypergeometric functions. The solution of the equation (68) for a large $|x|$ can be written as 


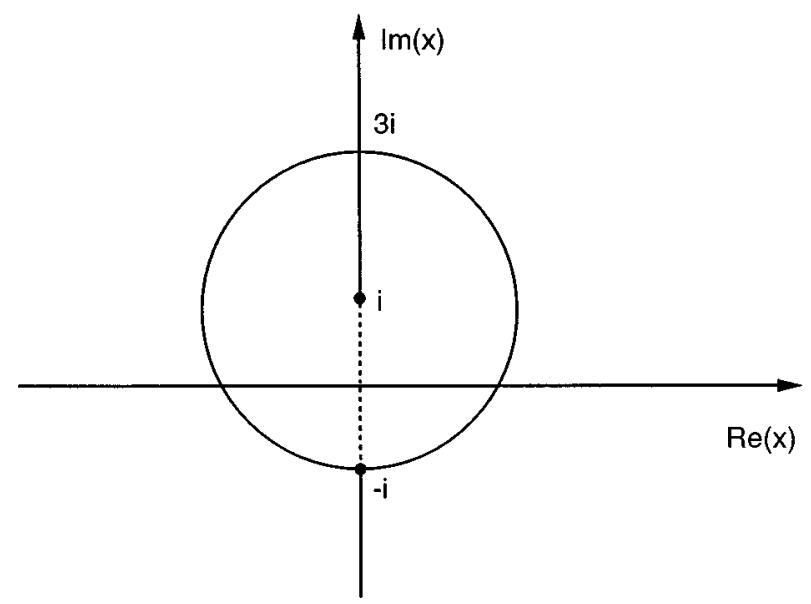

FIG. 1. Domain of convergence.

$$
\begin{aligned}
S(x)= & A_{1}(x-i)^{-(b+\mu) / 2-1 / 4}(x+i)^{b / 2+1 / 4}{ }_{2} F_{1}\left(\frac{a+b+1+\mu}{2}, \frac{b-a+1+\mu}{2} ; 1+\mu ; \frac{2 i}{i-x}\right) \\
& +A_{2}(x-i)^{-(b-\mu) / 2-1 / 4}(x+i)^{b / 2+1 / 4}{ }_{2} F_{1}\left(\frac{a+b+1-\mu}{2}, \frac{b-a+1-\mu}{2} ; 1-\mu ; \frac{2 i}{i-x}\right)
\end{aligned}
$$

with

$$
a^{2}=\left(b^{2}\right)^{*}=\frac{2 \beta^{2}-2 \alpha^{2}+1-i \gamma^{2}}{4}
$$

Let the separation constant $\mu$ be a positive number [the equation (71) is symmetric with respect to the replacement $\mu \rightarrow-\mu]$. Then the second term in formula (71) behaves like $|x|^{\mu / 2}$ at $\infty$ and must be omitted. Thus for $S(x)$ we obtain

$$
S(x)=A(x-i)^{-(b+\mu) / 2-1 / 4}(x+i)^{b / 2+1 / 4}{ }_{2} F_{1}\left(\frac{a+b+1+\mu}{2}, \frac{b-a+1+\mu}{2} ; \mu+1 ; \frac{2 i}{i-x}\right) .
$$

The hypergeometric function in Eq. (73) converges if $x$ lies out of the circle $C$ on Fig. 1, defined by $|i-x|=2$, and converges on the circle $C$ with the condition $\operatorname{Re}(b)<0$. The function $S(x)$ exists everywhere inside $C$ except the interval $x \in[-i, i]$, since the hypergeometric function in (73) has a cut along the argument $2 i /(i-x) \in[1, \infty)$. That means that the solution (73) along the real axes inside $C$ in general is not a continuous function and may have a jump at the point $x=0$. Let us now consider the analytic continuation of (73) inside the circle $C$ : 


$$
\begin{aligned}
S(x)= & A\left\{(x-i)^{a / 2+1 / 4}(x+i)^{b / 2+1 / 4} \frac{\Gamma(\mu+1) \Gamma(-a)}{\Gamma((b-a+1+\mu) / 2) \Gamma((-b-a+1+\mu) / 2)(2 i)^{(a+b+1+\mu) / 2}}\right. \\
& \cdot{ }_{2} F_{1}\left(\frac{a+b+1+\mu}{2}, \frac{a+b+1-\mu}{2} ; a+1 ; \frac{i-x}{2 i}\right) \\
& +(x-i)^{-a / 2+1 / 4}(x+i)^{b / 2+1 / 4} \frac{\Gamma(\mu+1) \Gamma(a)}{\Gamma((b+a+1+\mu) / 2) \Gamma((-b+a+1+\mu) / 2)(2 i)^{(-a+b+1+\mu) / 2}} \\
& \left.\cdot{ }_{2} F_{1}\left(\frac{-a+b+1+\mu}{2}, \frac{-a+b+1-\mu}{2} ;-a+1 ; \frac{i-x}{2 i}\right)\right\} .
\end{aligned}
$$

From Eq. (72) follow two possibilities

$$
a=b^{*}, \quad a=-b^{*} .
$$

Putting the $a=b^{*}[\operatorname{Re}(a)=\operatorname{Re}(b)<0]$ we find that the first term in (74) represents an analytic function, while the second term is discontinuous at $x=0$. [Note since the both terms in Eq. (74) transform to each other with replacement $a \rightarrow-a$, the choice $a=-b^{*}$ means that the first term in (74) is discontinuous while the second term is continuous at $x=0$.] Thus the sufficient condition for the existence of the continuous solution requires the relation

$$
\mu+a+a^{*}+1=-2 m, \quad m=0,1,2, \ldots,\left[-\frac{a+a^{*}+1}{2}\right],
$$

so from (72) we have

$$
\mu=-2 m-1+\frac{1}{\sqrt{2}} \sqrt{2 \beta^{2}-2 \alpha^{2}+1+\sqrt{\left(2 \beta^{2}-2 \alpha^{2}+1\right)^{2}+\gamma^{4}}} .
$$

Finally, the orthonormalized eigenfunction of Eq. (68) may be written in the form

$$
\begin{aligned}
S\left(\tau_{2}\right)= & (-1)^{3 m / 2} \Gamma(-a) \sqrt{\frac{\left(-2 m-a-a^{*}-1\right) \Gamma\left(-m-a^{*}\right)}{\pi m ! 2^{a+a^{*}+1} \Gamma(-m-a) \Gamma\left(-m-a-a^{*}\right)}} \\
& \cdot\left(1+i \sinh 2 \tau_{2}\right)^{a / 2+1 / 4}\left(1-i \sinh 2 \tau_{2}\right)^{a^{*} / 2+1 / 4} \\
& \cdot{ }_{2} F_{1}\left(-m, m+a+a^{*}+1 ; a+1 ; \frac{1+i \sinh 2 \tau_{2}}{2}\right) \\
= & (-1)^{m / 2} \sqrt{\frac{\left(-2 m-a-a^{*}-1\right) m ! \Gamma(-m-a) \Gamma\left(-m-a^{*}\right)}{\pi 2^{a+a^{*}+1} \Gamma\left(-m-a-a^{*}\right)}} \\
& \cdot\left(1+i \sinh 2 \tau_{2}\right)^{a / 2+1 / 4}\left(1-i \sinh 2 \tau_{2}\right)^{a^{*} / 2+1 / 4} P_{m}^{\left(a, a^{*}\right)}\left(-i \sinh 2 \tau_{2}\right),
\end{aligned}
$$

where

$$
\begin{aligned}
a= & \frac{1}{2^{3 / 2}}\left\{-\sqrt{\sqrt{\left(2 \beta^{2}-2 \alpha^{2}+1\right)^{2}+\gamma^{4}}+2 \beta^{2}-2 \alpha^{2}+1}\right. \\
& \left.+i \sqrt{\sqrt{\left(2 \beta^{2}-2 \alpha^{2}+1\right)^{2}+\gamma^{4}}-\left(2 \beta^{2}-2 \alpha^{2}+1\right)}\right\} .
\end{aligned}
$$

The second equation (69) is quite like (9) and has a solution 


$$
\begin{aligned}
Z\left(\tau_{1}\right) \equiv S_{n}^{(\alpha, \mu)}\left(\tau_{1}\right)= & \sqrt{\frac{2\left(\mu-\sqrt{2 \alpha^{2}+\frac{1}{4}}-2 n-1\right) \Gamma(\mu-n) n !}{\Gamma\left(\mu-\sqrt{2 \alpha^{2}+\frac{1}{4}}-n\right) \Gamma\left(1+n+\sqrt{2 \alpha^{2}+\frac{1}{4}}\right)}} \\
& \times\left(\sinh \tau_{1}\right)^{1 / 2+\sqrt{2 \alpha^{2}+1 / 4}}\left(\cosh \tau_{1}\right)^{1 / 2-\mu} P_{n}^{(\alpha,-\mu)}\left(\cosh 2 \tau_{1}\right)
\end{aligned}
$$

with $n=0,1, \ldots$.

The quantized energy is

$$
\begin{aligned}
E & =-\frac{1}{2}\left(\mu-\sqrt{2 \alpha^{2}+1 / 4}-2 n-1\right)^{2}+\frac{1}{8} \\
& =-\frac{1}{2}\left\{2 N+2+\sqrt{2 \alpha^{2}+\frac{1}{4}}-\frac{1}{\sqrt{2}} \sqrt{2 \beta^{2}-2 \alpha^{2}+1 \sqrt{\left(2 \beta^{2}-2 \alpha^{2}+1\right)^{2}+\gamma^{4}}}\right\}^{2}+\frac{1}{8},
\end{aligned}
$$

where $N=n+m$ is the principal quantum number and the bound state occurs for

$$
0 \leqslant N \leqslant\left[\frac{1}{\sqrt{8}} \sqrt{2 \beta^{2}-2 \alpha^{2}+1+\sqrt{\left(2 \beta^{2}-2 \alpha^{2}+1\right)^{2}+\gamma^{4}}}-\frac{1}{2} \sqrt{2 \alpha^{2}+\frac{1}{4}}-1\right] .
$$

The additional operator describing this coordinate system is

$$
\begin{aligned}
L_{1} \Psi_{n m}\left(\tau_{1}, \tau_{2}\right) & \equiv\left[K_{3}^{2}-2\left(\alpha^{2}-\beta^{2}\right)\left(\frac{\omega_{0}^{2}-\omega_{1}^{2}}{\omega_{0}^{2}+\omega_{1}^{2}}\right)^{2}-2 \gamma^{2} \frac{\omega_{0} \omega_{1}\left(\omega_{0}^{2}-\omega_{1}^{2}\right)}{\left(\omega_{0}^{2}+\omega_{1}^{2}\right)^{2}}\right] \Psi_{n m}\left(\tau_{1}, \tau_{2}\right) \\
& =\left\{2 m+1-\frac{1}{\sqrt{2}} \sqrt{2 \beta^{2}-2 \alpha^{2}+1+\sqrt{\left(2 \beta^{2}-2 \alpha^{2}+1\right)^{2}+\gamma^{4}}}\right\}^{2} \Psi_{n m}\left(\tau_{1}, \tau_{2}\right) .
\end{aligned}
$$

\section{Semi-hyperbolic coordinates}

Here

$$
\begin{gathered}
\omega_{0}^{2}=-\frac{\left(\mu-e_{3}\right)\left(\nu-e_{3}\right)}{2\left[\left(e_{3}-a\right)^{2}+b^{2}\right]}+\frac{1}{2}-\frac{1}{2 b}\left[\frac{\left[(\mu-a)^{2}+b^{2}\right]\left[(\nu-a)^{2}+b^{2}\right]}{\left(e_{3}-a\right)^{2}+b^{2}}\right]^{1 / 2}, \\
\omega_{1}^{2}=\frac{\left(\mu-e_{3}\right)\left(\nu-e_{3}\right)}{2\left[\left(e_{3}-a\right)^{2}+b^{2}\right]}-\frac{1}{2}-\frac{1}{2 b}\left[\frac{\left[(\mu-a)^{2}+b^{2}\right]\left[(\nu-a)^{2}+b^{2}\right]}{\left(e_{3}-a\right)^{2}+b^{2}}\right]^{1 / 2}, \\
\omega_{2}^{2}=-\frac{\left(\mu-e_{3}\right)\left(\nu-e_{3}\right)}{\left(e_{3}-a\right)^{2}+b^{2}}
\end{gathered}
$$

$\left[\nu<e_{3}<\mu\right]$, where $\sinh 2 f=\left(e_{3}-a\right) / b$ and $2 f$ is the distance between the focii of the semihyperbolas and the bases of their equidistants. ${ }^{6}$

If we change variables according to

$$
\omega_{0}=\frac{\left(s_{1}+s_{2}\right)}{\sqrt{2}}, \quad \omega_{1}=\frac{-i\left(s_{1}-s_{2}\right)}{\sqrt{2}}, \quad \omega_{2}=-i s_{3},
$$

the Schrödinger equation becomes 


$$
\begin{gathered}
\frac{1}{2}\left[\left(s_{1} \frac{\partial}{\partial s_{2}}-s_{2} \frac{\partial}{\partial s_{1}}\right)^{2}+\left(s_{1} \frac{\partial}{\partial s_{3}}-s_{3} \frac{\partial}{\partial s_{1}}\right)^{2}+\left(s_{3} \frac{\partial}{\partial s_{2}}-s_{2} \frac{\partial}{\partial s_{3}}\right)^{2}\right] \Psi \\
+\left[-E-\frac{1}{2}\left(\frac{k_{1}^{2}-\frac{1}{4}}{s_{1}^{2}}+\frac{k_{2}^{2}-\frac{1}{4}}{s_{2}^{2}}+\frac{k_{3}^{2}-\frac{1}{4}}{s_{3}^{2}}\right)\right] \Psi=0
\end{gathered}
$$

with

$$
\frac{1}{2}\left(k_{1}^{2}-\frac{1}{4}\right)=\frac{1}{4}\left(\beta^{2}-\alpha^{2}\right)-\frac{i}{8} \gamma^{2}, \quad \frac{1}{2}\left(k_{2}^{2}-\frac{1}{4}\right)=\frac{1}{4}\left(\beta^{2}-\alpha^{2}\right)+\frac{i}{8} \gamma^{2}, \quad \frac{1}{2}\left(k_{3}^{2}-\frac{1}{4}\right)=\alpha^{2}
$$

Noting

$$
\omega_{0}^{2}-\omega_{1}^{2}-\omega_{2}^{2}=s_{1}^{2}+s_{2}^{2}+s_{3}^{2}=1
$$

and considering Eq. (85), we see that the problem we wish to solve using the real coordinates $\omega_{0}$, $\omega_{1}$, and $\omega_{2}$ is a real case of the corresponding problem on the sphere with coordinates $s_{1}, s_{2}, s_{3}$ and energy $\varepsilon=-E$.

Inverting the relations $(84)$ we have

$$
s_{1}=\frac{\left(\omega_{0}+i \omega_{1}\right)}{\sqrt{2}}, \quad s_{2}=\frac{\left(\omega_{0}-i \omega_{1}\right)}{\sqrt{2}}, \quad s_{3}=i \omega_{2}
$$

Now choose elliptic coordinates on the complex sphere according to

$$
s_{1}^{2}=\frac{\left(\mu-e_{1}\right)\left(\nu-e_{1}\right)}{\left(e_{1}-e_{2}\right)\left(e_{1}-e_{3}\right)}, \quad s_{2}^{2}=\frac{\left(\mu-e_{2}\right)\left(\nu-e_{2}\right)}{\left(e_{2}-e_{1}\right)\left(e_{2}-e_{3}\right)}, \quad s_{3}^{2}=\frac{\left(\mu-e_{3}\right)\left(\nu-e_{3}\right)}{\left(e_{3}-e_{2}\right)\left(e_{3}-e_{1}\right)} .
$$

This choice of real coordinates $\mu, \nu$ will work for the real coordinates $\omega_{k}, k=0,1,2$, if we take $e_{1}=e_{2}^{*}=a+i b, a, b$ real and $\nu<e_{3}<\mu$.

In terms of the coordinates $\mu$ and $\nu$ the Schrödinger equation has the form

$$
\begin{aligned}
& \frac{4}{(\mu-\nu)}\left\{\left(\mu-e_{2}^{*}\right)\left(\mu-e_{2}\right)\left(\mu-e_{3}\right)\left[\frac{\partial^{2} \Psi}{\partial \mu^{2}}+\frac{1}{2}\left(\frac{1}{\mu-e_{2}^{*}}+\frac{1}{\mu-e_{2}}+\frac{1}{\mu-e_{3}}\right) \frac{\partial \Psi}{\partial \mu}\right]\right. \\
& \left.-\left(\nu-e_{2}^{*}\right)\left(\nu-e_{2}\right)\left(\nu-e_{3}\right)\left[\frac{\partial^{2} \Psi}{\partial \nu^{2}}-\frac{1}{2}\left(\frac{1}{\nu-e_{2}^{*}}+\frac{1}{\nu-e_{2}}+\frac{1}{\nu-e_{3}}\right) \frac{\partial \Psi}{\partial \nu}\right]\right\} \\
& +\left[\left(k_{1}^{2}-\frac{1}{4}\right) \frac{\left(e_{2}^{*}-e_{2}\right)\left(e_{2}^{*}-e_{3}\right)}{\left(\mu-e_{2}^{*}\right)\left(\nu-e_{2}^{*}\right)}+\left(k_{2}^{2}-\frac{1}{4}\right) \frac{\left(e_{2}-e_{2}^{*}\right)\left(e_{2}-e_{3}\right)}{\left(\mu-e_{2}\right)\left(\nu-e_{2}\right)}\right. \\
& \left.+\left(k_{3}^{2}-\frac{1}{4}\right) \frac{\left(e_{3}-e_{2}\right)\left(e_{3}-e_{2}^{*}\right)}{\left(\mu-e_{3}\right)\left(\nu-e_{3}\right)}\right] \Psi=-2 E \Psi .
\end{aligned}
$$

The separation equations are

$$
\begin{aligned}
& \left(\rho-e_{2}^{*}\right)\left(\rho-e_{2}\right)\left(\rho-e_{3}\right)\left[\frac{d^{2} \Psi}{d \rho^{2}}+\frac{1}{2}\left(\frac{1}{\rho-e_{2}^{*}}+\frac{1}{\rho-e_{2}}+\frac{1}{\rho-e_{3}}\right) \frac{d \Psi}{d \rho}\right] \\
& -\frac{1}{4}\left[\left(k_{1}^{2}-\frac{1}{4}\right) \frac{\left(e_{2}^{*}-e_{2}\right)\left(e_{2}^{*}-e_{3}\right)}{\left(\rho-e_{2}^{*}\right)}+\left(k_{2}^{2}-\frac{1}{4}\right) \frac{\left(e_{2}-e_{2}^{*}\right)\left(e_{2}-e_{3}\right)}{\left(\rho-e_{2}\right)}\right. \\
& \left.+\left(k_{3}^{2}-\frac{1}{4}\right) \frac{\left(e_{3}-e_{2}\right)\left(e_{3}-e_{2}^{*}\right)}{\left(\rho-e_{3}\right)}-2 E \rho+\lambda\right] \psi(\rho)=0,
\end{aligned}
$$


where $\rho=\mu, \nu$. The operator $L_{2}$ with eigenvalue $\lambda$ is

$$
\begin{aligned}
L_{2} \Psi= & \frac{-4}{(\mu-\nu)}\left\{\nu\left(\mu-e_{1}\right)\left(\mu-e_{2}\right)\left(\mu-e_{3}\right)\left[\frac{\partial^{2} \Psi}{\partial \mu^{2}}+\frac{1}{2}\left(\frac{1}{\mu-e_{2}^{*}}+\frac{1}{\mu-e_{2}}+\frac{1}{\mu-e_{3}}\right) \frac{\partial \Psi}{\partial \mu}\right]\right. \\
& -\mu\left[\left(\nu-e_{1}\right)\left(\nu-e_{2}\right)\left(\nu-e_{3}\right)\left[\frac{\partial^{2} \Psi}{\partial \nu^{2}}+\frac{1}{2}\left(\frac{1}{\nu-e_{2}^{*}}+\frac{1}{\nu-e_{2}}+\frac{1}{\nu-e_{3}}\right) \frac{\partial \Psi}{\partial \nu}\right]\right\} \\
& -\left[\left(k_{1}^{2}-\frac{1}{4}\right) \frac{\left(e_{2}^{*}-e_{2}\right)\left(e_{2}^{*}-e_{3}\right)}{\left(\mu-e_{2}^{*}\right)\left(\nu-e_{2}^{*}\right)}\left(\mu+\nu-e_{2}^{*}\right)+\left(k_{2}^{2}-\frac{1}{4}\right) \frac{\left(e_{2}-e_{2}^{*}\right)\left(e_{2}-e_{3}\right)}{\left(\mu-e_{2}\right)\left(\nu-e_{2}\right)}\left(\mu+\nu-e_{2}\right)\right. \\
& \left.+\left(k_{3}^{2}-\frac{1}{4}\right) \frac{\left(e_{3}-e_{2}\right)\left(e_{3}-e_{2}^{*}\right)}{\left(\mu-e_{3}\right)\left(\nu-e_{3}\right)}\left(\mu+\nu-e_{3}\right)\right] \Psi
\end{aligned}
$$

In order to find the bound state solutions of this system in semi-hyperbolic coordinates we first observe the identity

$$
\begin{aligned}
\frac{s_{1}^{2}}{\theta_{j}-e_{2}^{*}}+\frac{s_{2}^{2}}{\theta_{j}-e_{2}}+\frac{s_{3}^{2}}{\theta_{j}-e_{3}} & =\frac{\left(\omega_{0}^{2}-\omega_{1}^{2}\right)\left(\theta_{j}-a\right)-2 \omega_{0} \omega_{1} b}{\left(\theta_{j}-a\right)^{2}+b^{2}}-\frac{\omega_{2}^{2}}{\theta_{j}-e_{3}} \\
& =\frac{\left(\mu-\theta_{j}\right)\left(\nu-\theta_{j}\right)}{\left(\theta_{j}-e_{2}^{*}\right)\left(\theta_{j}-e_{2}\right)\left(\theta_{j}-e_{3}\right)} .
\end{aligned}
$$

If we then look for solutions of the form

$$
\Psi=\prod_{\ell=1}^{3} s_{\ell}^{k}+1 / 2 \prod_{j=1}^{N}\left(\frac{s_{1}^{2}}{\theta_{j}-e_{2}^{*}}+\frac{s_{2}^{2}}{\theta_{j}-e_{2}}+\frac{s_{3}^{2}}{\theta_{j}-e_{3}}\right),
$$

we see that the corresponding zeros satisfy the equations

$$
\frac{k_{1}+1}{\theta_{m}-e_{2}^{*}}+\frac{k_{2}+1}{\theta_{m}-e_{2}}+\frac{k_{3}+1}{\theta_{m}-e_{3}}+\sum_{j \neq m}^{N} \frac{2}{\left(\theta_{m}-\theta_{j}\right)}=0
$$

For the energy $E$ we have

$$
E=-\frac{1}{2}\left(2 N+2+k_{1}+k_{2}+k_{3}\right)^{2}+\frac{1}{8},
$$

which coincides with the formula (73) [note (86)]. For the separation constant $\lambda$ we obtain

$$
\begin{aligned}
\lambda= & -2\left[k_{1}\left(e_{2}+e_{3}\right)+k_{2}\left(e_{2}^{*}+e_{3}\right)+k_{3}\left(e_{2}+e_{2}^{*}\right)\right]-2\left[e_{3} k_{1} k_{2}+e_{2} k_{1} k_{3}+e_{2}^{*} k_{2} k_{3}\right] \\
& -\frac{3}{2}\left(e_{2}^{*}+e_{2}+e_{3}\right)-4 e_{2} e_{3}\left(k_{1}+1\right) \sum_{m=1}^{q} \frac{1}{\left(\theta_{m}-e_{2}^{*}\right)}-e_{2}^{*} e_{3}\left(k_{2}+1\right) \sum_{m=1}^{q} \frac{1}{\left(\theta_{m}-e_{2}\right)} \\
& -4 e_{2} e_{2}^{*}\left(k_{3}+1\right) \sum_{m=1}^{q} \frac{1}{\left(\theta_{m}-e_{3}\right)} .
\end{aligned}
$$

In terms of variables $w_{i}$ the total wave function is written

$$
\Psi=\left(\frac{\omega_{0}+i \omega_{1}}{\sqrt{2}}\right)^{k_{1}+1 / 2}\left(\frac{\omega_{0}-i \omega_{1}}{\sqrt{2}}\right)^{k_{2}+1 / 2}\left(i \omega_{2}\right)^{k_{3}+1 / 2} \prod_{j=1}^{N}\left[\frac{\left(\omega_{0}^{2}-\omega_{1}^{2}\right)\left(\theta_{j}-a\right)-2 \omega_{0} \omega_{1} b}{\left(\theta_{j}-a\right)^{2}+b^{2}}-\frac{\omega_{2}^{2}}{\theta_{j}-e_{3}}\right] .
$$

The algebra of second-order symmetries for this potential is generated by the operators

$$
L_{j k}=\left(s_{j} \partial_{s_{k}}-s_{k} \partial_{s_{j}}\right)^{2}+\left(\frac{1}{4}-k_{j}^{2}\right) \frac{s_{k}^{2}}{s_{j}^{2}}+\left(\frac{1}{4}-k_{k}^{2}\right) \frac{s_{j}^{2}}{s_{k}^{2}}
$$


for $j, k=1,2,3$ and $j \neq k$. The Hamiltonian of the system is expressed in terms of $L_{j k}$ as

$$
H=\frac{1}{2}\left(L_{12}+L_{13}+L_{23}\right)-\frac{1}{2} \sum_{i=1}^{3} k_{i}^{2}+\frac{3}{4} .
$$

The relevant generators in the real case we are considering are then

$$
\begin{aligned}
L_{12}=-K_{3}^{2}+\left(\frac{1}{4}-k_{1}^{2}\right)\left(\frac{\omega_{0}-i \omega_{1}}{\omega_{0}+i \omega_{1}}\right)^{2}+\left(\frac{1}{4}-k_{2}^{2}\right)\left(\frac{\omega_{0}+i \omega_{1}}{\omega_{0}-i \omega_{1}}\right)^{2}, \\
L_{13}=\frac{1}{2}\left(M_{1}-i K_{2}\right)^{2}+\left(\beta^{2}-\alpha^{2}-\frac{i}{2} \gamma^{2}\right) \frac{\omega_{2}^{2}}{\left(\omega_{0}+i \omega_{1}\right)^{2}}+\alpha^{2} \frac{\left(\omega_{0}+i \omega_{1}\right)^{2}}{\omega_{2}^{2}}, \\
L_{23}=\frac{1}{2}\left(M_{1}+i K_{2}\right)^{2}+\left(\beta^{2}-\alpha^{2}+\frac{i}{2} \gamma^{2}\right) \frac{\omega_{2}^{2}}{\left(\omega_{0}-i \omega_{1}\right)^{2}}+\alpha^{2} \frac{\left(\omega_{0}-i \omega_{1}\right)^{2}}{\omega_{2}^{2}} .
\end{aligned}
$$

The commutation relations and resulting quadratic algebra can then be deduced from the relations for the complex forms in terms of the $L_{i j}$. It is easy to show that the additional integrals of motion, corresponding to the separation in equidistant and semi-hyperbolic coordinates, can be written as

$$
L_{1}=-L_{12}+\beta^{2}-\alpha^{2}
$$

and

$L_{2}=e_{3} L_{12}+e_{2} L_{13}+e_{1} L_{32}-k_{1}^{2}\left(e_{2}+e_{3}-e_{1}\right)-k_{2}^{2}\left(e_{1}+e_{3}-e_{2}\right)-k_{3}^{2}\left(e_{1}+e_{2}-e_{3}\right)+\frac{1}{4}\left(e_{1}+e_{2}+e_{3}\right)$.

The algebra for the operators (99) and (100) is found in Ref. 11.

\section{ACKNOWLEDGMENTS}

The authors thank Professor V. M. Ter-Antonyan and Professor P. Winternitz for interesting discussions.

${ }^{1}$ C. P. Boyer, E. G. Kalnins, and P. Winternitz, "Completely integrable relativistic Hamiltonian systems and separation of variables in Hermitian hyperbolic spaces," J. Math. Phys. 24, 2022 (1983)

${ }^{2}$ C. Grosche, G. S. Pogosyan, and A. N. Sissakian, "Path integral approach to superintegrable potentials. The twodimensional hyperboloid,' Phys. Part. Nuclei 27, 244 (1996).

${ }^{3}$ E. G. Kalnins, W. Miller, Jr., and G. S. Pogosyan, "Superintegrability on the two dimensional hyperboloid," J. Math. Phys. 38, 5416 (1997).

${ }^{4}$ P. Winternitz, I. Lukac, and Ya. A. Smorodinskii, "Quantum numbers in the little groups of the Pioncare group," Sov. J. Nucl. Phys. 7, 139 (1968).

${ }^{5}$ E. G. Kalnins and W. Miller, Jr., "Lie theory and separation of variables, 4: The groups $\mathrm{SO}(2,1)$ and $\mathrm{SO}(3)$," J. Math. Phys. 15, 1263 (1974).

${ }^{6} \mathrm{M}$. N. Olevskiĭ, "Triorthogonal systems in spaces of constant curvature in which the equation $\Delta_{2} u+\lambda u=0$ allows the complete separation of variables," Math. Sb. 27, 379 (1950) (in Russian).

${ }^{7}$ L. D. Landau and E. M. Lifshitz, Quantum Mechanics: Non-relativistic Theory (Pergamon, Oxford, 1977).

${ }^{8}$ Higher Transcendental Functions edited by A. Erdélyi, W. Magnus, F. Oberhettinger, and F. G. Tricomi (McGraw-Hill, New York, 1953), Vols. I and II.

${ }^{9}$ A. Frank and K. B. Wolf, "Lie Algebras for systems with mixed spectra. I. The scattering Pöschl-Teller potential," J. Math. Phys. 26, 973 (1985).

${ }^{10}$ A. F. Nikiforov, S. K. Suslov, and V. B. Uvarov, Classical Orthogonal Polynomials of Discrete Variables (Nauka, Leningrad, 1985)

${ }^{11}$ E. G. Kalnins, W. Miller, Jr., and G. S. Pogosyan, “Superintegrability and associated polynomial solutions. Euclidean space and the sphere in two dimensions," J. Math. Phys. 37, 6439 (1996). 